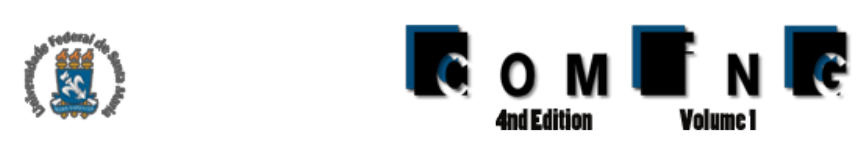

\title{
Ferramenta para monitoramento de redes wireless com foco em amenizar a aglomeração de usuário em pontos de acesso
}

\author{
Thales Nicolai Tavares ${ }^{1}$, Renato Preigschadt Azevedo ${ }^{2}$ \\ ${ }^{1}$ Universidade Federal de Santa Maria (UFSM) \\ CEP - 91.105-900 - Santa Maria - RS - Brasil \\ \{tavares, renato\}@redes.ufsm.br
}

\begin{abstract}
By monitoring the network, you can improve the quality of service in order to increase efficiency and productivity. This work describes a tool developed called CTIWifi, which used PHP libraries together with information collections from the network, the tool that aims to assist in wireless network monitoring, presenting easy-to-read graphics of infrastructure information.
\end{abstract}

Resumo. O monitoramento da rede, pode-se melhorar a qualidade do serviço, tendo em vista aumentar a eficiência e produtividade. Este trabalho descreve uma ferramenta desenvolvida denominada CTIWifi, a qual utilizado bibliotecas PHP junto com coletas de informações da rede, a ferramenta que tem como objetivo auxiliar no monitoramento da rede sem fio apresentando gráficos de fácil leitura das informações da infraestrutura.

\section{Introdução}

As redes de computadores foram criadas com o propósito inicial de compartilhamento de dados e serviços de dispositivos. De acordo com [Kurose and Ross 2010], quando o número de usuários de redes de computadores não era muito grande, a gerência e o monitoramento não eram utilizados. Porém, com o passar do tempo, a rápida evolução das tecnologias de redes foi o fator que contribuiu para a difusão das redes de computadores pelo mundo todo.

Segundo [Stallings 1999], as redes de computadores, que não possuem gerenciamento, estão sujeitas a não continuar funcionando por muito tempo. O gerenciamento e monitoramento permitem a construção de uma representação geral e em tempo real do estado da rede para o seu administrador. Isto permite a prevenção e detecção de problemas na infraestrutura das redes de computadores, provendo assim uma operação com maior qualidade.

Gerenciamento de redes de computadores é o controle de atividades e monitoramento dos elementos da rede. Através do monitoramento da rede, é possível melhorar a qualidade de serviço, tendo em vista a eficiência e produtividade. A prática de gerenciamento de redes de computadores está tornando-se uma tarefa difícil, especialmente em redes constituídas por muitos dispositivos.

A utilização de ferramentas que possibilitam o gerenciamento de redes está se tornando cada vez maior. Levando em consideração que a tarefa de gerenciar uma rede de computadores pode ser composta por um ou mais serviços, trabalhando em conjunto para monitorar as possíveis falhas que comprometam sua integridade. 
Ferramenta para monitoramento de redes wireless com foco em amenizar

Para permitir um monitoramento mais eficiente e um maior acesso as informações, foi desenvolvida uma ferramenta de monitoramento com o foco em redes sem fio denominada CTIWifi. Tendo em vista detectar problemas, esta ferramenta busca auxiliar os administradores de redes na tarefa de monitoramento, apresentando gráficos de fácil leitura das informações da infraestrutura sem fio. A ferramenta recebeu este nome em homenagem ao Colégio Técnico Industrial de Santa Maria, onde o curso de Redes de Computadores esta vinculado. Essa ferramenta foi desenvolvida em PHP (Hypertext PreProcessor) devido conceitos básicos da linguagem ser de fácil de aprendizagem, além de possuir uma grande quantidade de bibliotecas disponíveis, o que facilita no desenvolvimento de aplicações.

\section{Background}

Atualmente, é possível realizar o monitoramento dos dispositivos em uma rede devido à protocolos de gerenciamento, os quais facilitam a tarefa de monitorar os dispositivos existentes na rede. Segundo [Dias 2008] os protocolos de monitoramento de rede determinam um formato para o envio de dados entre os ativos de redes monitorados e as máquinas responsáveis pelo armazenamento de tais informações.

\subsection{Protocolo SNMP}

O SNMP (Simple Network Management Protocol) surgiu no final da década de 80 para complementar a necessidade de um modelo para gerenciar redes TCP/IP. Para [Tanenbaum 2003] o SNMP é um protocolo de gerência típica das redes TCP/IP, que pertence à camada de aplicação, e sua função é facilitar a troca de informações entre os dispositivos de rede, facilitando o gerenciamento de uma rede.

Segundo [Tanenbaum 2003] os dispositivos gerenciados possuem um agente SNMP instalado. Esses dispositivos coletam e armazenam dados do gerenciamento e disponibilizam estas informações para o sistema de gerenciamento. Os dispositivos monitorados podem ser roteadores, servidores, computadores, switches entre outros dispositivos de redes.

O SNMP segue o modelo Gerente - Agente, onde um computador funciona como Gerente e os demais funcionam como agentes, podendo dessa forma comunicar-se com o dispositivo de rede analisado. De acordo com [Mauro and Schmidt 2005] é possível gerenciar qualquer dispositivo que possibilite a recuperação de informações compatíveis com o protocolo SNMP.

As operações do SNMP tem por propósito a troca de informações entre o gerente e agente, sobre os objetos gerenciados no ativo monitorado. De acordo com [Moqadi and da Silva 2011] uma das características do protocolo SNMP é ser simples, pois dispõe de um conjunto básico de operações que é baseado no paradigma conhecido como busca-armazenamento, ou seja, que as operações do protocolo SNMP são derivadas de operações básicas de busca e armazenamento.

\subsection{MIB - Base de informação gerenciável}

O SMI (Structure of Management Information) proporciona uma maneira de definir os objetos gerenciados e seus comportamentos. Um agente possui uma lista de objetos que 
Ferramenta para monitoramento de redes wireless com foco em amenizar

ele controla. Um possível objeto é o status operacional, como exemplo o status da interface de um computador que pode estar up ou down. Podemos pensar em um banco de dados de objetos gerenciados e rastreados pelos agentes onde qualquer tipo de status ou dados estatísticos que podem ser acessados pelo gerente, esta base de dados recebe o nome de MIB (Management Information Base). A MIB é utilizada para administrar os elementos em uma rede de computadores. Na maior parte das vezes associadas com o Protocolo SNMP, o termo também é utilizado de forma genérica em contextos como no modelo OSI (Open Systems Interconnection).

Para [Kurose and Ross 2010] a base de informações de gerenciamento é quase um banco de dados virtual que preserva em seus objetos os dados coletados da rede. As informações são coletadas pelos dispositivos gerenciados ou pelo gerente SNMP. As informações estão organizadas na MIB de forma hierárquica. Segundo [Kurose and Ross 2010] somente as folhas da árvore, isto é, os nós das extremidades são os objetos gerenciados. Qualquer objeto representa uma informação capturada do dispositivo, como por exemplo, informações do consumo da rede.

A hierarquia de uma MIB pode ser compreendida como uma árvore de raiz anônima, possuindo seus galhos distribuídos por diferentes organizações. A camada mais alta das OID (Object IDentifier) da MIB é padronizada por diferentes organizações, enquanto a camada mais baixa é alocada em organizações associadas. Essa arquitetura possibilita a gestão em todas as camadas do modelo de referência OSI. Assim as MIBs podem ser estabelecidas para cada área específica de informação e operação, e é possível estender as aplicações e informações monitoradas.

\section{Trabalhos Relacionados}

O trabalho desenvolvido por [Kurose and Ross 2010], apresenta uma análise da possibilidade e a necessidade de implementação de um aplicativo para o monitoramento de redes Wireless, aplicadas ao provimento de serviços de internet. Neste trabalho é utilizado o protocolo de gerência SNMP para obtenção de dados sobre as condições da rede, a linguagem de programação Java para o desenvolvimento do aplicativo, e o serviço WEB para permitir a interface com o usuário final.

O autor descreve que o estudo efetuado no ambiente de transmissão sem fio, o elemento Wireless Bridge (WavePOINT-II) foi pesquisado, e neste foi identificado o suporte a gerência de redes via SNMP. Segundo [Pisching 2001], este equipamento é o principal elemento para a comunicação sem fio, o qual faz a ligação entre a base de transmissão e as estações remotas no cenário de seu trabalho. O autor percebe neste equipamento a necessidade de monitoramento a fim de permitir controlar o seu funcionamento.

As interfaces desenvolvidas [Pisching 2001] estão divididas em módulos, e cada um deles possui um objetivo específico. As interfaces desenvolvidas permitem este monitoramento, através da WEB, como mostra a Figura 1, a qual apresenta informações sobre as interfaces de rede.

As interfaces desenvolvidas neste trabalho permitem o monitoramento através da WEB, como foi apresentado na Figura 1, que demostra a interface do módulo "Dados sobre Interface", a qual apresenta ao administrador as informações sobre as interfaces da entidade gerenciada. Como pode-se observar, são listadas as interfaces identificadas, 


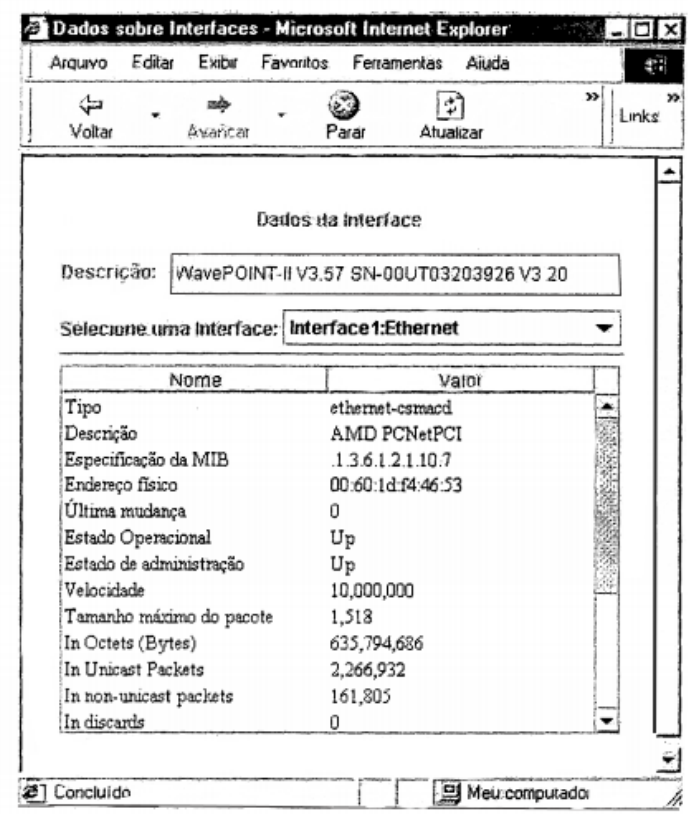

Figura 1. Tela do módulo Dados sobre as Interfaces.

e, quando uma delas é selecionada em particular, são apresentadas diversas informações coletadas sobre a interface.

Outra funcionalidade desta ferramente é o módulo "Dados da tabela IP ARP”, conforme mostra a Figura 2, a qual apresenta uma simples tabela que segundo o autor, onde cada linha corresponde a uma interface física do sistema. As informações em cada linha fornecem um mapeamento do endereço de rede para endereço físico.

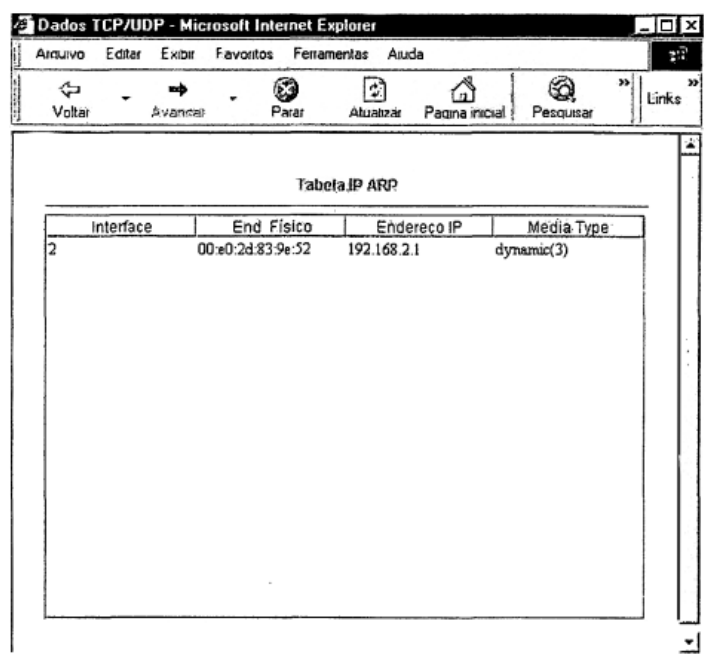

Figura 2. Tela do módulo Dados da tabela IP ARP.

Conforme [Pisching 2001], com os valores coletados e apresentados através das interfaces implementadas, o autor chegou à conclusão de que o administrador de uma rede sem fio pode tomar decisões sobre seus equipamentos Wireless, quando estes apresenta- 
Ferramenta para monitoramento de redes wireless com foco em amenizar

rem algum resultado inadequado.

No trabalho proposto por [Santos 2011], é apresentado um software de monitoramento para roteadores Wireless, onde o mesmo utiliza-se do protocolo SNMP para realizar a comunicação com roteadores que tenham o firmware DD-WRT ou OpenWrt. Se os roteadores da rede a ser monitorada tenham o firmware DD-WRT ou OpenWrt e possua o serviço de SNMP habilitado, a ferramenta desenvolvida pode se conectar a estes equipamentos e ler informações como taxa de utilização de uma determinada interface, endereço MAC (Media Access Control) das interfaces, quantidade de usuários conectados a este roteador, entre outras.

Através das informações obtidas nesta ferramenta o administrador da rede pode identificar, por exemplo, qual ponto de acesso está sendo mais utilizado e se este ponto está sobrecarregado. As informações apresentadas na ferramenta são distribuídas na tela em três seções, sendo uma com informações do roteador, outra com informações das interfaces do roteador, sendo possível navegar pelas interfaces e por fim uma seção com informações dos clientes Wireless conectados, também com possibilidade de escolher de qual cliente se deseja mostrar as informações, como mostra a Figura 3.

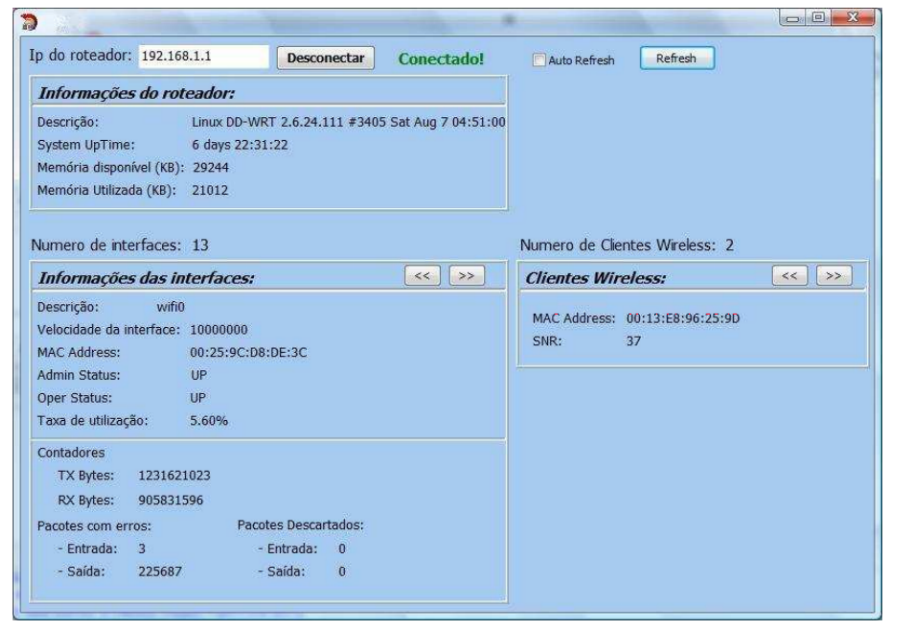

Figura 3. Tela do software de monitoramento.

A ferramenta de monitoramento foi elaborada em linguagem de programação $\mathrm{C}++$. Para o seu desenvolvimento foi usada uma versão Trial do Borland $\mathrm{C}++$ Builder. $\mathrm{O}$ Borland $\mathrm{C}++$ Builder dispõe uma série de componentes para as mais diversas finalidades. Para a comunicação SNMP com os roteadores foi usado o componente TidSNMP para deixar transparente ao programador toda a troca de mensagens do protocolo até que se consiga executar uma operação SNMP.

O autor do trabalho afirma que o software precisa de melhorias para se tornar um sistema de larga utilização, porém o que foi implementado até então prova que este sistema como um todo pode ser muito útil.

Existem ferramentas de acordo com os trabalhos relacionados, quais foram desenvolvidas para o monitoramento voltado para redes sem fio. Porém não possuem gráficos compreensíveis de informações da rede sem fio.

Os trabalhos apresentados, possuem em comum a intenção de apoiar e incentivar 
a utilização de ferramentas de gerenciamento e monitoramento de redes. Por este motivo, este trabalho apresenta a ferramenta CTIWifi, a qual irá auxiliar o monitoramento de redes Wireless.

\section{Desenvolvimento}

O funcionamento da aplicação é apresentando em blocos ligados entre si que representam, por sua vez, os módulos da arquitetura funcional da aplicação e a transferência da informação por ela tratada, como mostra a Figura 4.

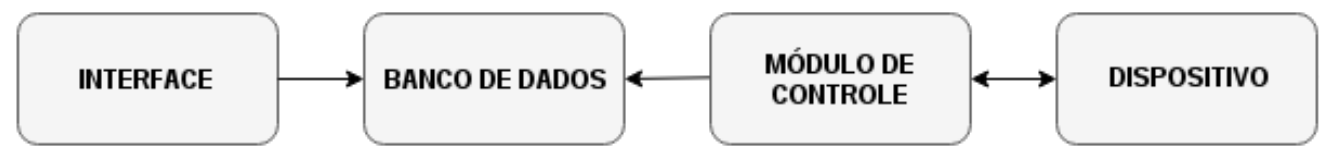

Figura 4. Diagrama Funcional.

Em termos gerais, podemos descrever os módulos da seguinte forma. Interface responsável pela representação gráfica da informação manipulada pela aplicação e que permite a utilização desta por parte do utilizador, nela foram utilizadas as linguagens JavaScript,HTML5 e CSS. O Banco de Dados é responsável por guardar a informação e retorná-la quando solicitada por parte da interface. O módulo de Controle possui responsabilidade de executar as ações de obtenção da informação do dispositivo e tratar para posteriormente ser armazenada. Por fim, temos os dispositivos que são os elementos da rede a serem monitorados pela aplicação.

No que diz respeito à arquitetura na qual se dispõe o funcionamento da aplicação, está pode ser ilustrada pela Figura 5. Como se pode verificar, os dispositivos monitorados estão ligados através da rede, onde se executam os módulos de controle e armazenamento. A informação é coletada, tratada e armazenadas em uma base de dados no servidor, onde todos os pedidos de dados são solicitados pela interface, através do servidor WEB.

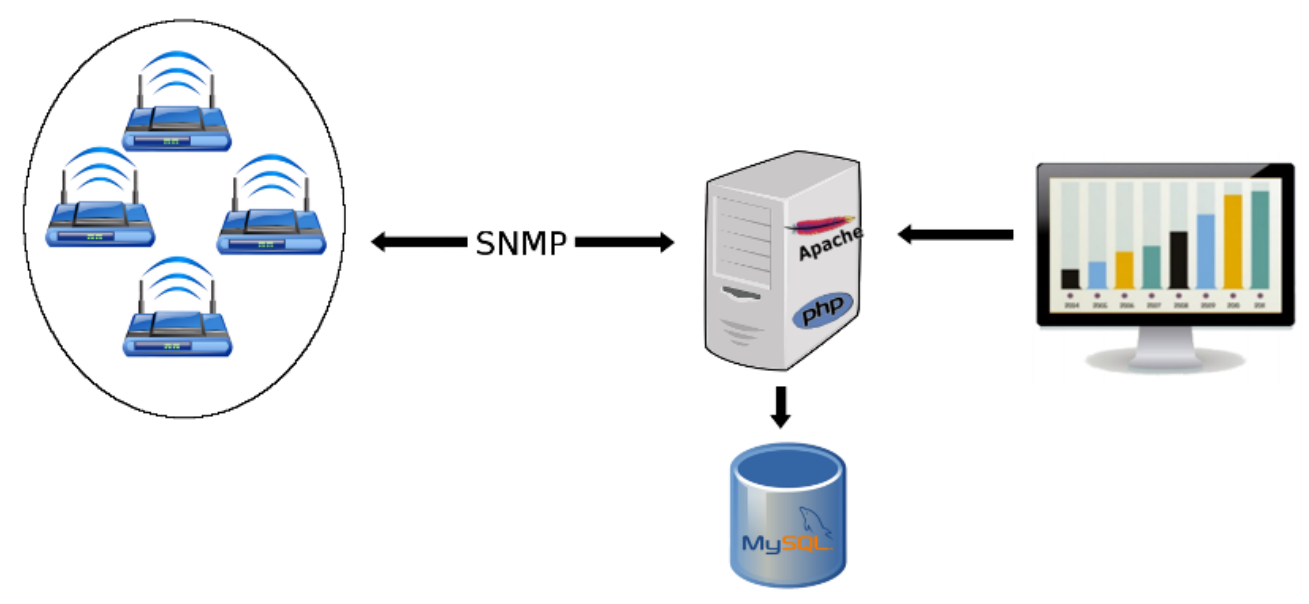

Figura 5. Arquitetura da Aplicação. 
Ferramenta para monitoramento de redes wireless com foco em amenizar

\subsection{Módulo de Controle}

Para realização da tarefa de coletar e armazenar as informações dos Access Points, foi criada uma classe denominada MostraConectados que possui dois métodos, sendo eles chamados de conectadosShow e salvaConectados, como mostra a Figura 6. Esses métodos serão responsáveis por realizar busca e armazenamento das informações.

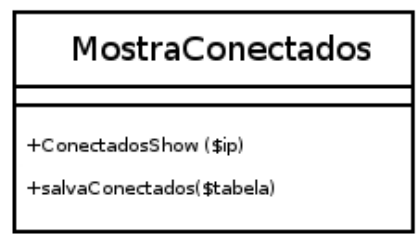

Figura 6. Classe MostraConectados.

O método conectadosShow, que é responsável por realizar a busca das informações, recebe como entrada uma lista contendo os endereços IP's dos roteadores sem fio. Logo é percorrido todos os equipamentos e são consultados os sensores relevantes em busca de informação da quantidade de usuários conectados, bem como velocidade e potência. Após coletar as informações, é realizado o processo de tratamento e depois enviado para armazenamento no banco de dados.

Para realizar o armazenamento das informações, após receber o devido tratamento é executado o outro método existente nessa classe chamado de salvaConectados. Esse método recebe a informação de qual a tabela do banco de dados deve ser armazenada, e com o auxilio de outro método pertencente a classe banco é executada a tarefa de armazenamento das informações, para uso futuro.

\subsection{Classe Banco}

desenvolvida uma classe que é responsável por manipular o banco de dados. A classe Banco, possui métodos para realizar conexão com o banco, inserção, consulta, através de uma consulta SQL e método para finalizar a conexão, como mostra a Figura 7. Esta classe possui alguns atributos, as quais contém informação sobre o endereço do banco de dados, bem como a base de dados e informações para autenticação.

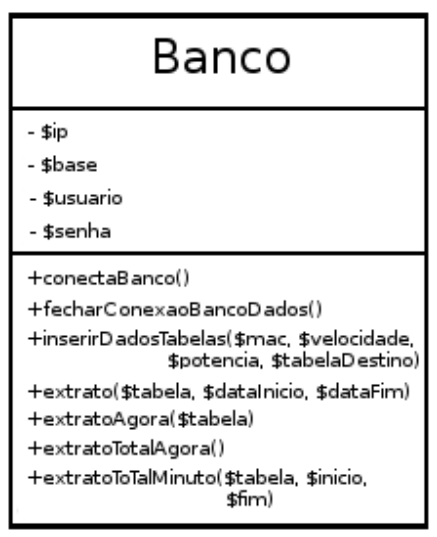

Figura 7. Classe Banco. 
Ferramenta para monitoramento de redes wireless com foco em amenizar

O método conectaBanco é o responsável por estabelecer a conexão com o banco de dados. Esse método é utilizado quando ocorre alguma forma de manipulação das tabelas do banco de dados apresentada na Figura 8. O método inserirDadosTabelas é utilizado para realizar a tarefa de salvar os dados tratados na classe MostraConectados. Para inserir os dados na tabela tabela DEVICE, o método recebe como parâmetros, as informações como $M A C$, velocidade, potência.

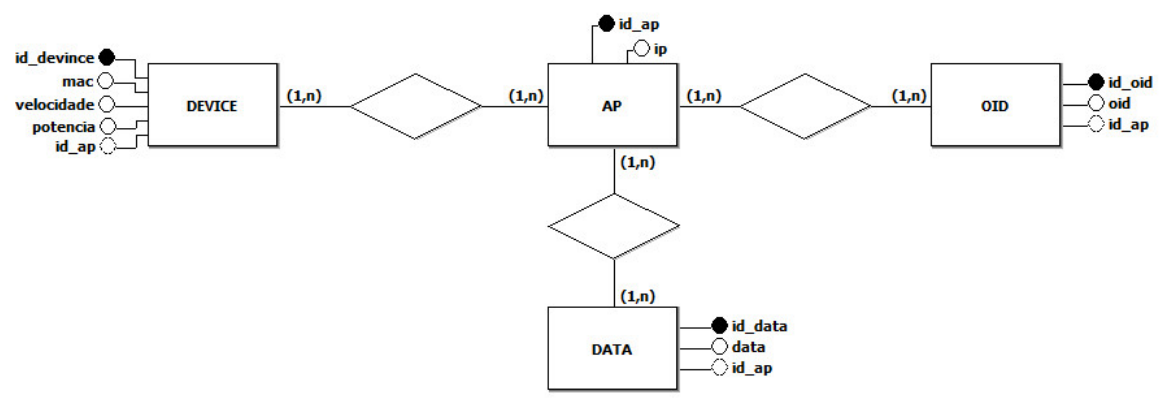

Figura 8. Modelo ER do banco.

Para realizar a busca das informações existentes no banco de dados, são utilizados os métodos extrato, extratoAgora, extratoTotalAgora. Estes métodos realizam a busca das informações desejadas, conforme o gráfico que se deseja. O método extrato é utilizado para buscar no banco de dados a quantidade de dispositivos conectados, e esse método necessita que seja passado argumentos para ele, informando em qual tabela fará a busca e o período de início e fim.

Para saber a quantidade de dispositivos conectados atualmente em um Access Point, é necessário a utilização do método extratoAgora. Esse método é informado em qual tabela devera realizar a busca da quantidade de dispositivos que estejam conectados, sendo assim, trazendo a informação da quantidade de dispositivos conectados em um determinado Access Point. Para saber a quantidade de dispositivos conectados por minuto, é utilizado o método extratoToTalMinuto, o qual possibilita ter um conhecimento da quantidade máxima por minuto conectado.

Para que seja possível informar a quantidade de dispositivos conectados em toda a rede, é utilizado o método extratoTotalAgora. Esse método tem a função de fazer uma busca no banco de dados da quantidade total dos dispositivos conectados, ou seja, fará uma pesquisa em todas as tabelas e retornara um somatório dos resultados obtidos.

\subsection{Interface}

A interface gráfica é quem permite mostrar ao administrador da rede as informações coletadas. Para essa etapa foi desenvolvido arquivos com a estrutura de uma página HTML5 necessários para a criação dos gráficos de forma a ser genéricos, ou seja, de forma que possam se adaptar conforme as informações coletadas do banco de dados. Neste módulo foi usado a biblioteca Chart.JS, que permite a visualização de dados através de gráficos diversos e interativos. A biblioteca utiliza elemento canvas do HTML5 para desenhar esses gráficos em uma página WEB.

Para gerar os gráficos, é realizado o acesso a classe Banco e seus métodos. Através da chamada desses métodos, o script contido no arquivo que gera os gráficos realiza uma 
consulta na tabela que foi passada. Após a consulta é gerado o gráfico e exibido na tela, como mostra a Figura 9.

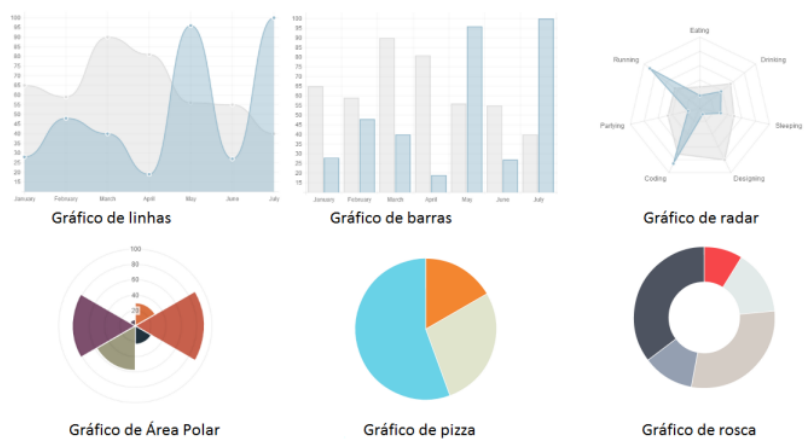

Figura 9. Gráficos da Interface.

\section{Experimentos e Resultados}

Para realizar o teste da ferramenta CTIWifi, foi monitorada a rede Wireless disponibilizada pelo Colégio Técnico Industrial de Santa Maria, onde a ferramenta foi inserida em uma máquina virtual do VMS (sistema de máquina virtuais), disponibilizada pela instituição. Este sistema busca auxiliar no ensino das diversas disciplinas ministradas neste ambiente estudantil, buscando auxiliar nas atividades empregadas. Essa máquina virtual possui o sistema operacional Ubuntu 12.04.1 LTS (Long Term Support), onde foi instalado um servidor WEB Apache, no qual foram hospedadas as páginas PHP desenvolvidas.

No momento em que tentamos acessar o endereço da ferramenta através de um navegador de internet, surge a página de início de sessão solicitando um usuário e senha válidos para acesso a ferramenta, como mostra a Figura 10. Será necessário informar as credenciais de acesso para poder utilizar a aplicação, essas credenciais serão verificadas encontram-se presente no banco de dados.

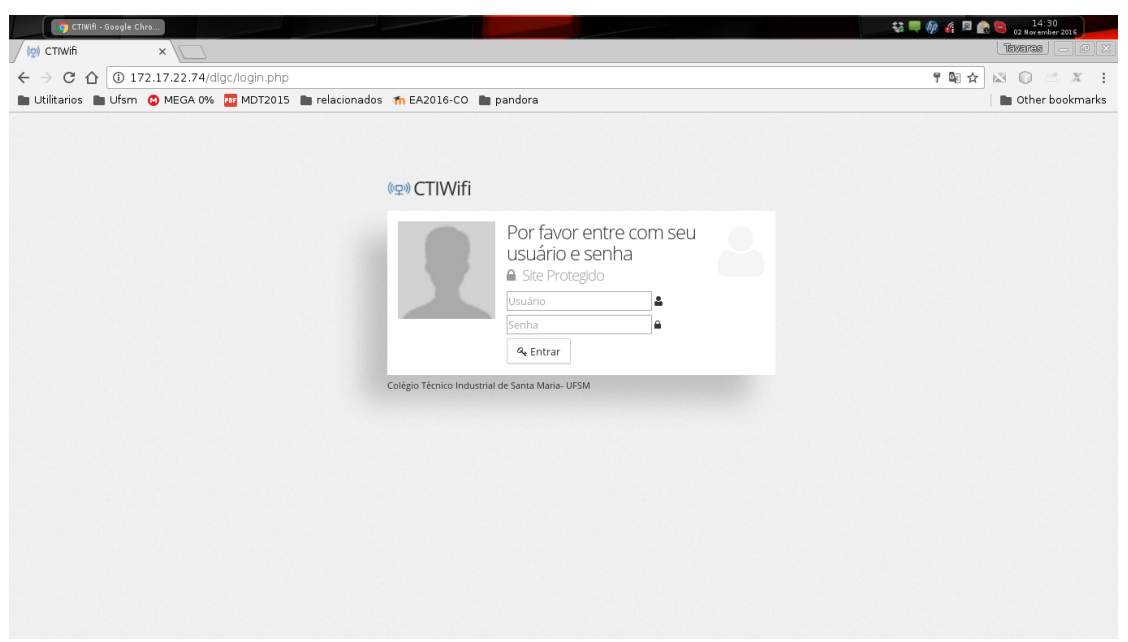

Figura 10. Tela de Login da Aplicação. 
Ferramenta para monitoramento de redes wireless com foco em amenizar

pós se autenticar-se na ferramenta, pode ser realizada a busca dos Access Point que serão monitorados pela ferramenta, como mostra a Figura 11. Para realizar a busca do dispositivos, foi informado a faixa de endereço IP dos dispositivo.

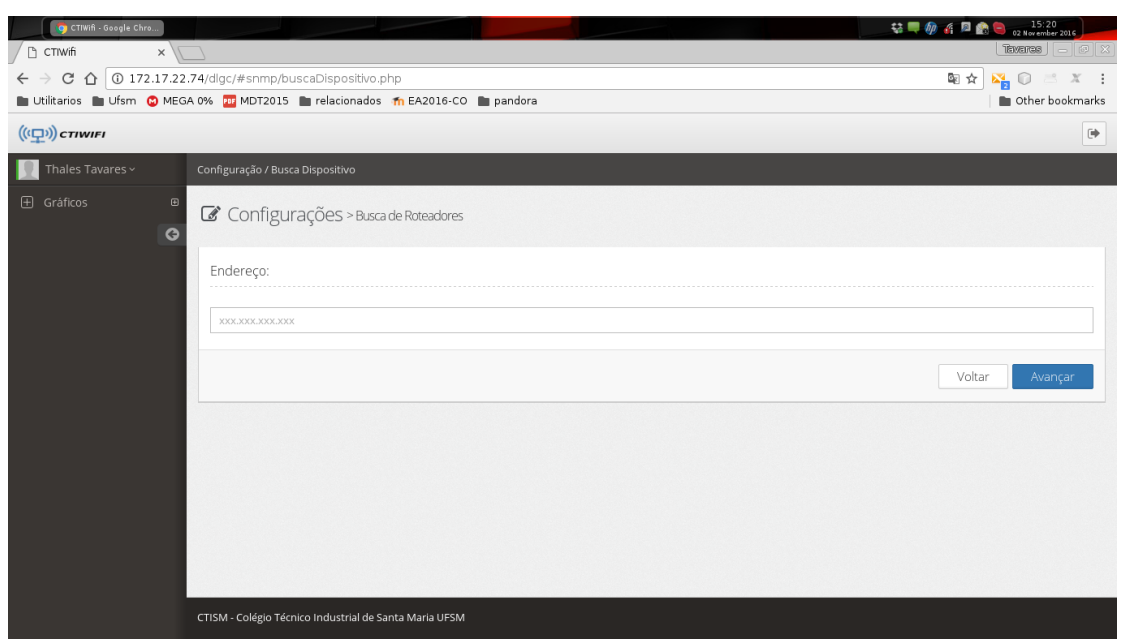

Figura 11. Tela de Busca de Dispositivos.

Será necessario informar a versão do protocolo SNMP que está sendo utilizado nos dispositivos, como mostra a Figura 12. No caso da rede Wireless do Colégio Técnico Industrial de Santa Maria, foi selecionado a segunda versão do protocolo SNMP e informado a string de comunidade public, a qual é utilizada nos dispositivos da rede.

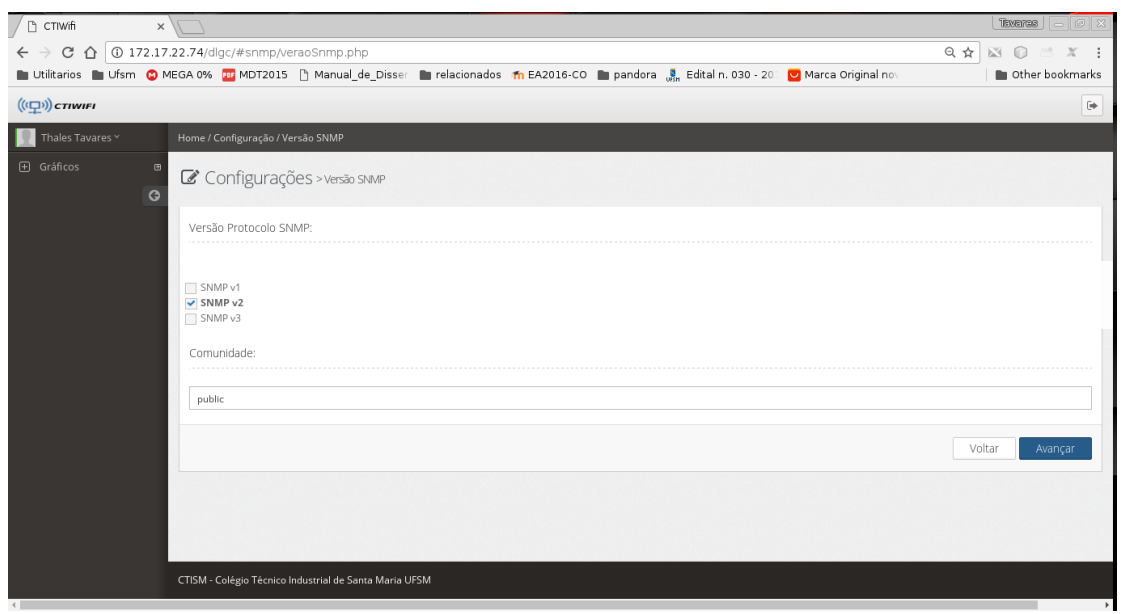

Figura 12. Tela de Seleção da versão do protocolo SNMP.

Logo após ser realizada a busca dos Access Point a serem monitorados, é retornado uma tela com os dispositivos encontrados dentro da faixa de endereço IP informada anteriormente, conforme a Figura 13. A tela apresentada informações o nome cadastrado no dispositivo, endereço IP, local cadastrado no dispositivo e o fabricante.

O administrador da rede deve selecionar os dispositivos que serão monitorados. Isso permite evitar que a ferramenta insira algum dispositivo desnecessário. Logo após selecionar os dispositivos, o administrador deve informar qual a quantidade total de dispositivos conectados em cada Access Point, sendo que essas informações serão armazenadas 
Ferramenta para monitoramento de redes wireless com foco em amenizar a aglomeração de usuário em pontos de acesso

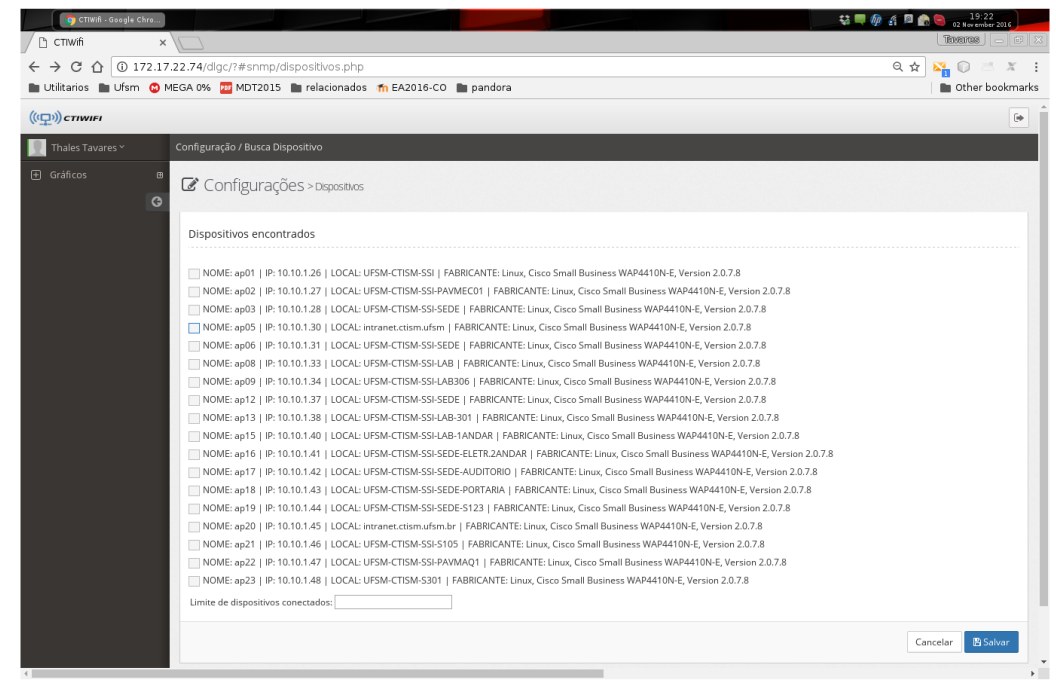

Figura 13. Tela de Dispositivos Encontrados.

no banco de dados. O próximo passo é configurar o e-mail que receberá as notificações de alertas, como mostra a Figura 14.

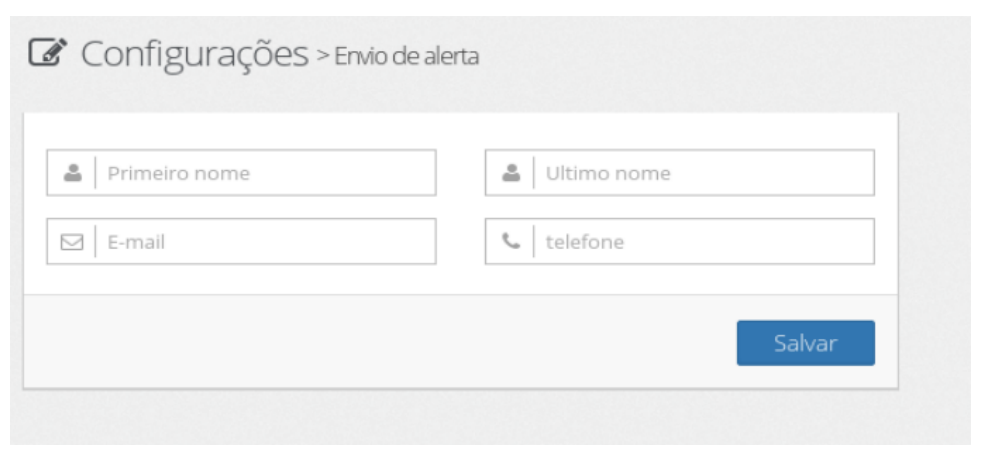

Figura 14. Tela de Configuração de e-mail para Alerta.

Ao terminar as configurações, a ferramenta constrói um menu na parte lateral onde se apresentam os equipamentos monitorados na rede, como mostra a Figura 15. Esse menu é montado pela ferramenta a partir das informações que foram armazenadas no banco de dados anteriormente, quando foi realizado a busca dos dispositivos.

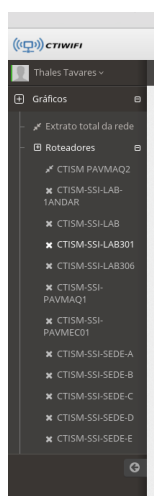

Figura 15. Menu de Navegação da Aplicação. 
Ferramenta para monitoramento de redes wireless com foco em amenizar

Com a ferramenta configurada, os gráficos da quantidade de usuário conectados na rede sem fio começa a ser gerados pela aplicação. Os gráficos exibem informações sobre a quantidade de dispositivos conectado durante os dias do mês corrente, um gráfico mostrando o histórico do ano corrente, conforme a Figura 16.

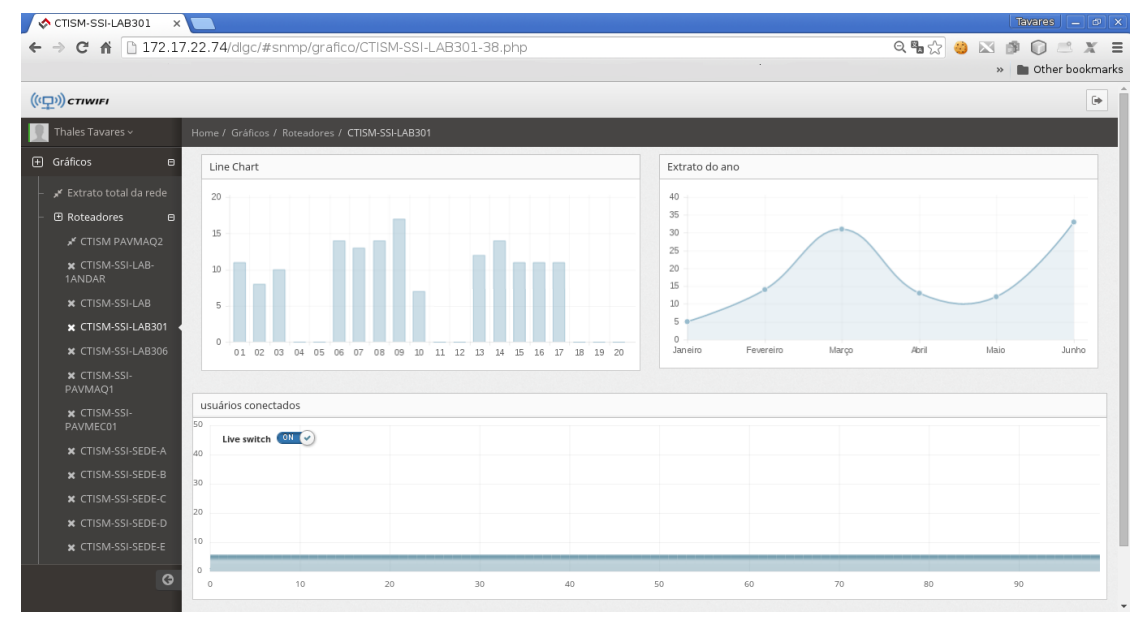

Figura 16. Gráficos referente ao Access Point CTISM-SSI-LAB301.

Os gráficos mostram a quantidade de dispositivos conectados naquele Access Point, sendo atualizado constantemente. Através do menu que encontra-se na lateral da interface, tem-se a possibilidade de escolher qual Access Point deseja exibir os gráficos.

Os gráficos exibidos na figura acima referem-se aos dispositivos que conectaram no Access Point CTISM-SSI-LAB301. Para cada Access Point é gerado um modo de visualização diferente ou desejado, isso fica a cargo do adminstrador da rede e da ferramenta, como mostra a Figura 18, que exibe as informações do Access Point CTISM-SSILAB-1ANDAR. A ferramenta CTIWifi permite, além de uma visualização segmentada, o gráfico que mostra a quantidade de dispositivos totais conectados. Este gráfico possui atualizações constantes, buscando ter uma precisão exata da quantidade de dispositivos conectados.

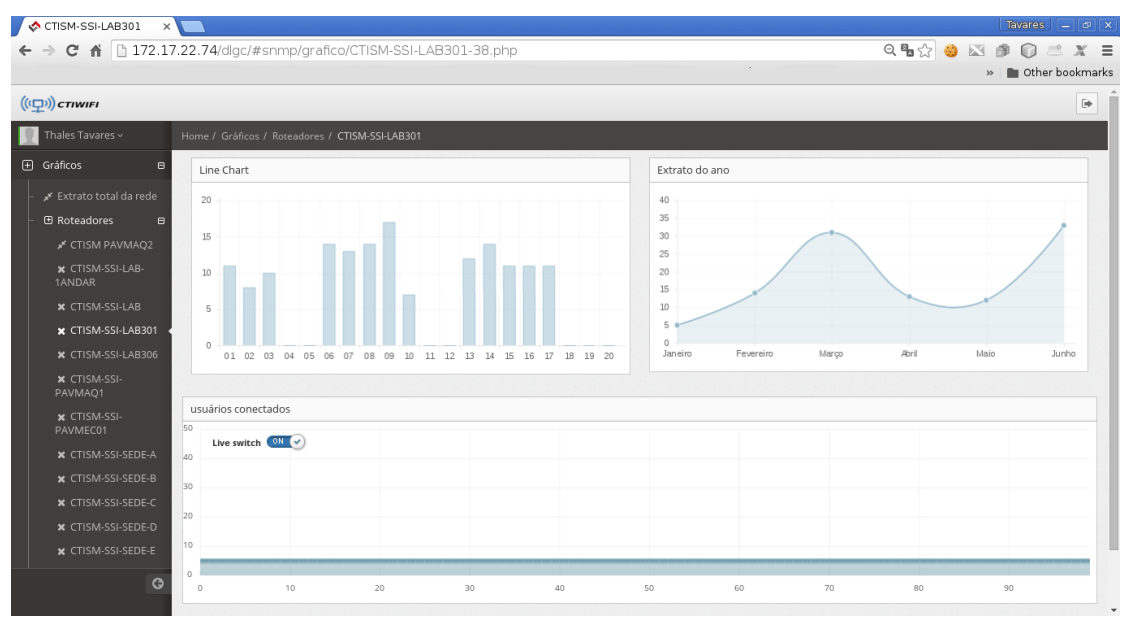

Figura 17. Gráficos referente ao Access Point CTISM-SSI-LAB-1ANDAR.

Quando a quantidade de usuário conectados em um Acess Point está acima do 
Ferramenta para monitoramento de redes wireless com foco em amenizar

configurado, o gráfico de usuário conectados troca sua cor para vermelho. Vemos na Figura 18, que a troca da cor do gráfico é para mostrar ao administrador de rede que o Access Point CTISMSSI-SEDE-A esta com a quantidade de usuários conectados acima do configurado.

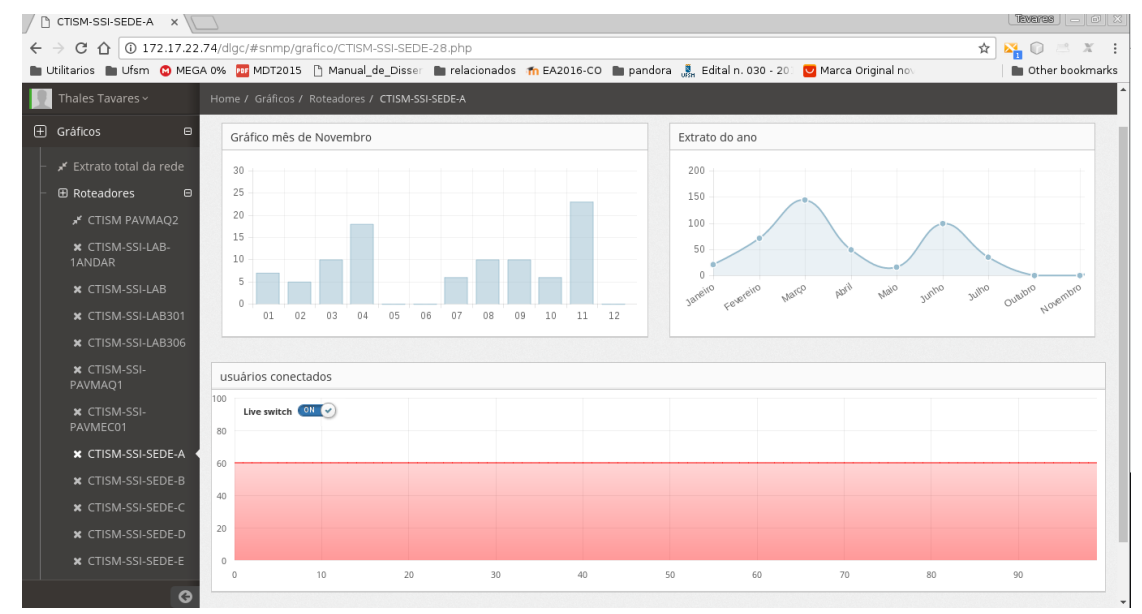

Figura 18. Gráficos referente ao Access Point CTISM-SSI-SEDE-A.

O gráfico altera sua cor para vermelho quando ultrapassa a quantidade de usuário conectados. Quando isso ocorre, é enviado um e-mail de alerta para o endereço de contato configurado anteriormente na ferramenta. Essa medida é tomada para que tenha uma garantia maior que o administrador da rede seja informado do ocorrido, para que não seja preciso estar em frente a ferramenta durante todo o tempo.

\section{Considerações finais}

O gerenciamento e monitoramento de redes de computadores é uma área que requer trabalho e dedicação, tanto para visão acadêmica como empresarial. Devido a sua grande granularidade, essa área é composta vários protocolos, inúmeras maneiras de se monitorar dispositivos, vários serviços e softwares que podem auxiliar o administrador de redes.

Com o crescimento das redes de computadores sem fio, um sistema de gerenciamento de redes torna-se cada vez mais indispensável. Possuir as informações corretas, possibilita aos administradores de rede tomar decisões coerente e em tempo hábil, minimizando assim indisponibilidade de equipamentos ou serviço.

A proposta apresentada neste trabalho demonstrou resultados satisfatórios, considerando que atendeu ao objetivo proposto. Através da implementação da ferramenta de monitoramento, constatou-se que a solução proposta é viável e que sua utilização pode auxiliar os administradores de redes.

Com as informações coletadas e apresentadas através da ferramenta desenvolvida, chegasse à conclusão que a utilização da mesma pode dar ao administrador da rede informações importantes. Com essas informações, o administrador pode tomar decisões sobre seus equipamentos Wireless quando estes apresentarem algum resultado inadequado. Estas decisões poderão ser tomadas, através da análise dos dados referentes a quantidade de dispositivos presentes na rede, locais em que estão. Sendo assim, o administrador pode realizar uma ação proativa, como por exemplo, aumento de capacidade ou 
Ferramenta para monitoramento de redes wireless com foco em amenizar

área de cobertura em determinados pontos da rede. Essas decisões que serão tomadas, serão com base nas informações apresentadas pela ferramenta.

Como proposta de trabalhos futuros poderão ser implementadas novas funcionalidades, como por exemplo, descoberta automática de novos Access Point através da verificação de rede. Sendo que hoje a busca de dispositivos é realizada manualmente no momento da configuração da ferramenta. Outra sugestão é integração da ferramenta com o servidor de autenticação RADIUS, para que seja possível identificar os usuários conectados por Access Point. Também será melhorado o aspecto da escolha do protocolo SNMP, o qual será realizada a descoberta automática da versão protocolo utilizada pelo dispositivo.

\section{Referências}

Dias, H. d. L. (2008). A importância do monitoramento de ativos de redes: Um estudo de caso com o sistema cacic.

Kurose, J. F. and Ross, K. W. (2010). Redes de Computadores e a Internet: Uma abordagem top-down. Pearson, São Paulo, SP, trad. 5 ed. edition.

Mauro, D. and Schmidt, K. (2005). Essential SNMP. O’Reilly Media, 2 edition.

Moqadi, K. A. A. and da Silva, V. C. O. (2011). Uso de ferramentas de gerencia de rede para analise de desempenho de uma rede local. Universidade Luterana do Brasil (Ulbra).

Pisching, M. A. (2001). Uma aplicação java-snmp para monitoramento de redes sem fio.

Santos, C. J. M. d. (2011). Sistema de monitoramento para redes sem fio.

Stallings, W. (1999). SNMP, SNMPv2, SNMPv3, and RMON 1 and 2. Addison-Wesley, 3 edition.

Tanenbaum, A. S. (2003). Redes de Computadores. Campus, Rio de Janeiro, RJ, 3 edition. 